\title{
Prospectiva post pandemia COVID-19 para elaborar el planteamiento del problema de los proyectos de investigación en las ciencias administrativas
}

\author{
Prospective post pandemic COVID-19 to elaborate the \\ approach to the problem of research projects in \\ administrative sciences
}

\section{Luis Fernando Valeriano} Ortiz

lvalerianoo@unmsm.edu.pe

Universidad Nacional Mayor de San Marcos, Facultad de Ciencias Administrativas. Lima, Perú

Presentado: 25/02/2021 - Aceptado: 01/03/2021 - Publicado: 17/06/2021

(c) Los autores. Este artículo es publicado por la revista Gestión en el Tercer Milenio de la Facultad de Ciencias Administrativas Universidad Nacional Mayor de San Marcos. Este es un artículo de acceso abierto, distribuido bajo los términos de la licencia Creative Commons Atribución 4.0 Internacional (CC BY 4.0) [https://creativecommons.org/licenses/by/4.0/deed.es] que permite el uso, distribución y reproducción en cualquier medio, siempre que la obra original sea debidamente citada de su fuente original. 
para el desarrollo de los proyectos de investigación dentro del análisis de su macro entorno en la problemática de su estudio.

Palabras claves: Prospectiva; pandemia; COVID-19.

\begin{abstract}
As of November 2019, countries worldwide are affected by a pandemic, known as coronavirus 19 , the ravages of which are evident in the fact that health systems are overwhelmed by the great demand for care from patients infected by this virus, recently In December 2020 a light emerges in the tunnel with positive results with the development of a vaccine in the most developed countries, but which unfortunately will arrive in Peru in 2021. This summarized description of the global health problem has collateral effects on development of economies, in the exponential growth of vulnerable populations, in the deterioration of the educational system at all levels, in the citizen insecurity fueled by drug addiction and in our country an unprecedented political crisis is added among the powers of the state generating greater instability and increased country risk, in the same way, research projects under development are affected by the uncertainty factor of the effects of this pandemic. Peru by 2021 requires a new political order through the elections of new authorities, which guarantees it to have governmental stability, strengthening the democratic system, developing policies that combat corruption and immorality on all fronts, this scourge is worse than covid 19 due to its implications and effects on Peruvian society. Peru is an ancient country, with a wealth of natural resources throughout the national territory, in the coast, mountains and jungle and with an entrepreneurial people, this real scenario allows me to affirm that the country will achieve economic recovery in the short term term, once a safe and efficient vaccine is applied to the Peruvian population. Therefore, this article has a perspective vision and aims to identify the strengths and opportunities that the country has to achieve its recovery, which will serve as a guide for the development of research projects within the analysis of its macro environment in the problem of your study.
\end{abstract}

Keywords: Prospective; pandemic; COVID-19.

\section{INTRODUCCIÓN}

El primer infectado de COVID-19 que llega el Perú fue en marzo del 2019, el Gobierno tomó medidas drásticas como cerrar las fronteras, confinamiento de la población en sus domicilios a nivel nacional por tres meses, se paralizó la economía nacional, etc., solamente se aseguró el abastecimiento de los productos de primera necesidad, pero los resultados no fueron los esperados, creció exponencialmente la cantidad de personas infectadas desbordando la capacidad de atención hospitalaria pública y privada en el ámbito nacional, este apretado resumen motivo que los sectores sociales como la salud, educación y trabajo se deterioren afectando principalmente a las poblaciones vulnerables de menores recursos, de igual forma los sectores productivos y de servicios colapsen por falta de inversiones y los sistemas financieros entren a un periodo recesivo y especulativo. Muchas investigaciones básicas y aplicadas se paralizaron o sufrieron distorsiones muy severas en su desarrollo.

El Perú es más grande que sus propios problemas coyunturales y estructurales, como se demuestra a la largo de su historia, cuenta con una población preparada para superar los problemas generados por la naturaleza o por el hombre, su riqueza de recursos naturales a lo largo y ancho del territorio nacional le garantizan una recuperación sostenible en el tiempo y las nuevas generaciones de peruanos constituyen la reserva moral del país, en consecuencia tenemos que tener una visión prospectiva para garantizar su reactivación y desarrollo, sobre la base de la disciplina, moral, equidad y justicia social dentro de un sistema democrático, estas condiciones de reactivación harán posible que 
se continúen con las investigaciones puras o básicas y aplicadas.

\section{OBJETO DEL ARTÍCULO}

Realizar un análisis reflexivo de la realidad nacional como consecuencia de la pandemia COVID-19 y plantear una visión prospectiva del desarrollo sectorial cuando la población peruana cuente con una vacuna segura y efectiva, serán la base para la elaboración del planteamiento del problema en las investigaciones.

\section{ARGUMENTACIÓN}

El artículo describe algunos aspectos estratégicos restrictivos que afectan el macro entorno del país debido a la pandemia COVID-19 y su visión prospectiva de desarrollo del Perú, que deberían considerarse en el planteamiento del problema en el desarrollo de las investigaciones propuestas por los estudiantes de post y pre grado de la Facultad de Ciencias Administrativas de la UNMSM, aplicando el método deductivo (de lo general a lo particular) y los enfoques cuantitativos, cualitativos o mixtos. A continuación, se identifican los principales factores y algunos indicadores que deberían considerarse en la problemática de la investigación según corresponda:

\section{Aspectos Regulatorios o Legales}

La Constitución Política del Perú es el marco legal que norma la estructura política, económica y social del país, tiene como fin promover su desarrollo. El Perú cumple 200 años de vida republicana en el 2021, ha tenido 12 Constituciones Políticas, en estas últimas décadas se acentúa una crisis política que amerita reformas constitucionales según juristas y constitucionalistas. El problema de fondo es el interés de la clase política por cambiar el capítulo económico de la Constitución de 1993, que genera amplios debates por juristas y políticos, a mi criterio sería un error cambiar del modelo de una economía social de mercado por una economía planificada. Sin embargo, la realidad nacional demanda el cambio constitucional en muchos de los capítulos de la actual Constitución.

\section{Aspectos Políticos}

El factor más relevante de los aspectos políticos es el riesgo país, que se refiere al grado de estabilidad política de una nación con respecto a las inversiones, en la medida que aumente la inestabilidad política de un país, los inversores retraen sus inversiones por el nivel de riesgo que están asumiendo. El riesgo país debe ser analizado sobre la base de cuatro aspectos:

- Riesgo político, evalúa los conflictos políticos internos como externos.

- Riesgo nación, cuando se decide no cumplir sus compromisos de su deuda externa e interna.

- Riesgo de liquidez, el país deudor no pueda asumir sus compromisos financieros.

- Riesgo macroeconómico, no se está en la capacidad de asumir su deuda debido a problemas coyunturales y estructurales de su macroeconomía y microeconomía.

\section{Aspectos Económicos}

El régimen económico del país se sustenta en la economía social de mercado, en el pluralismo económico, en la libre competencia y en la libertad de contratar, y en los principales acuerdos comerciales que tiene el Perú, como es la Comunidad Andina de Naciones (CAN), Mercado Común del Sur (Mercosur) y el Foro de Cooperación Económica Asia-Pacifico (APEC).

La Figura 1 refleja que durante las últimas décadas el comportamiento del Producto Bruto Interno (PBI) en el Perú siempre tuvo resultados positivos con sus altas y bajas hasta el 2019. El deterioro acelerado de la economía peruana es como consecuencia de la pandemia a partir del periodo 2020 .

\section{Las Proyecciones para el 2021}

Según la Comisión Económica para América Latina y el Caribe (CEPAL, 2021) se pronostica que "la región experimentaría un repunte del crecimiento del 3,7\% en promedio. América del Sur crecería un 3,7\%, Centroamérica y México, un $3,8 \%$, y el Caribe, un 4,2\%" (p. 116). En la Figura 2 , se puede apreciar que la economía peruana crecería 9\% en el 2021.

CEPAL (2020) afirma que: 
Figura 1

Variación Porcentual del PBI del Perú en los últimos años previos a la pandemia.

PERÚ: OFERTA Y DEMANDA GLOBAL 2007 - 2019

(Variación porcentual del indice de volumen fisico) Mho Base $2007=100$

\begin{tabular}{|c|c|c|c|c|c|c|c|c|c|c|c|c|c|}
\hline Oferta y Demanda Global & 2007 & 2008 & 2009 & 2010 & 2011 & 2012 & 2013 & 2014 & 2015 & 2016P/ & 2017P/ & 2018E/ & $2019 \varepsilon /$ \\
\hline Producto Bruto interno & 8,5 & 9,1 & 1,1 & 8,3 & 6,3 & 6,1 & 5,9 & 2,4 & 3,3 & 4,0 & 2,5 & 4,0 & 2,2 \\
\hline Extractivas & 4,1 & 7,9 & 0,5 & 1,3 & 2,8 & 1,8 & 4,3 & $-1,4$ & 6,9 & 9,0 & 3,4 & 1,9 & 0,2 \\
\hline Transformación & 11,9 & 10,5 & $-3,2$ & 12,0 & 7,0 & 5,3 & 6,5 & $-0,2$ & $-2,3$ & $-0,9$ & 1,1 & 5,6 & $-0,7$ \\
\hline Servicios ${ }^{1 /}$ & 9,0 & 9,1 & 3,0 & 9,5 & 7,3 & 7,8 & 6,1 & 4,4 & 4,1 & 4,1 & 2,7 & 4,1 & 3,6 \\
\hline Importadiones & 21,3 & 25,0 & $-15,9$ & 26,6 & 13,6 & 10,0 & 2,9 & $-1,0$ & 1,0 & 1,6 & 7,1 & 3,3 & 1,3 \\
\hline Oferta y Demanda Global & 10,8 & 12,2 & $-2,6$ & 11,7 & 7,9 & 7,0 & 5,2 & 1,6 & 2,8 & 3,4 & 3,5 & 3,8 & 2,0 \\
\hline Demanda Interna & 12,3 & 13,7 & $-2,4$ & 14,5 & 8,6 & 8,1 & 6,7 & 3,0 & 2,4 & 1,5 & 2,1 & 3,8 & 2,4 \\
\hline Consumo Final Privado & 8,6 & 8,9 & 3,1 & 9,1 & 7,2 & 7,4 & 5,7 & 4,2 & 4,0 & 3,7 & 2,6 & 3,7 & 3,0 \\
\hline Consumo del Gobierno & 4,3 & 4,8 & 12,1 & 3,9 & 7,4 & 8,3 & 7,5 & 6,5 & 8,0 & 5,2 & 3,3 & 2,7 & 4,5 \\
\hline Formación Bruta de Capital & 28,6 & 31,1 & $-20,2$ & 35,8 & 12,2 & 9,5 & 8,5 & $-1,1$ & $-3,6$ & $-5,8$ & 0,0 & 4,7 & $-0,3$ \\
\hline Formación Bruta de Capital Fijo & 22,7 & 27,8 & $-3,4$ & 21,9 & 9,9 & 14,9 & 5,4 & $-2,0$ & $-7,2$ & $-4,5$ & 1,3 & 4,7 & 2,9 \\
\hline Público & 20,4 & 33,7 & 29,7 & 16,3 & $-11,3$ & 19,8 & 12,3 & 1,1 & $-5,7$ & 0,1 & $-3,9$ & 6,5 & $-0,8$ \\
\hline Privado & 23,2 & 26,5 & $-11,0$ & 23,7 & 16,6 & 13,7 & 3,6 & $-2,9$ & $-7,6$ & $-5,8$ & 2,9 & 4,2 & 4,0 \\
\hline Exportadiones & 6,7 & 7,8 & $-3,3$ & 3,2 & 5,5 & 3,1 & $-0,6$ & $-3,8$ & 4,3 & 11,8 & 8,8 & 3,9 & 0,5 \\
\hline
\end{tabular}

Nota. Tomado del Instituto Nacional de Estadística e Informática, 2020.

Figura 2.

América Latina y el Caribe (33 países): proyecciones de crecimiento, 2021 (en porcentajes)

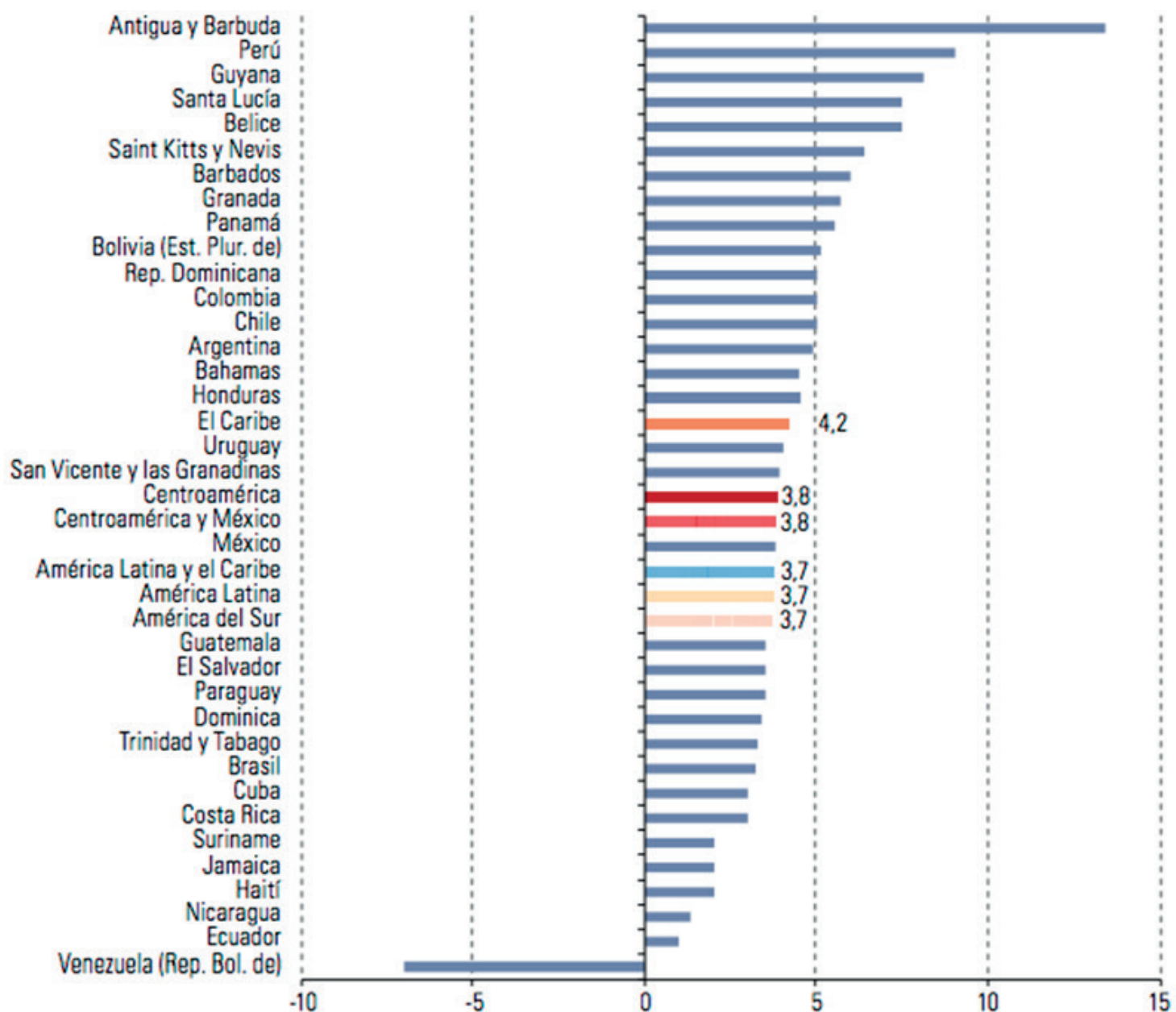

Nota. Tomado de Balance Preliminar de las Economías de América Latina y el Caribe 2020 (p. 117), por CEPAL, 2020. 
Prospectiva post pandemia COVID-19 para elaborar el planteamiento del problema de los proyectos de investigación en las...

en reiteradas ocasiones, el papel de las políticas macroeconómicas activas es crucial para que, con posterioridad al rebote que podría ocurrir en 2021, la región logre dinamizar su crecimiento más allá de lo que ha sido su desempeño en los últimos años. (p. 117)

\section{Los Sectores Productivos y de Servicios Estratégicos}

Constituyen las líneas funcionales del desarrollo socio-económico del país y podrían ser materia de análisis en las investigaciones.

\section{Sector minero}

El Perú se ubica entre los primeros productores de diversos minerales a nivel mundial (oro, plata, cobre, plomo, zinc, hierro, estaño, molibdeno, teluro, entre otros).

El yacimiento de litio encontrado en el sur del país, puede ser procesado e industrializado es un insumo muy requerido en el mundo para el avance tecnológico y está vinculado a la industria eléctrica para elaborar baterías.
La situación actual de los proyectos mineros en el Perú se puede observar en la Figura 3, donde se muestra 46 proyectos que se encuentran en etapa de desarrollo.

\section{La agroindustria}

El Perú es muy rico en la agroindustria, en productos tales como palta, uva, espárrago y arándano, el Gobierno Central tiene que mejorar el acceso al agua y debe promoverse el inicio de proyectos.

En la Tabla 1 se detalla el porcentaje de variación que se obtuvo frente al año anterior en el mismo período.

\section{Sector pesquero industrial}

Es un gran dinamizador de la economía, aporta con $1,5 \%$ al PBI en forma directa y genera alrededor de 700.000 empleos.

Pesca ilegal señala que las embarcaciones foráneas entran a puerto peruano a recibir mantenimiento, lo que les facilita la navegación y extracción cerca de nuestro mar, falta una política de estado que fortalezca la conservación

\section{Figura 3}

Cartera de proyectos de construcción de mina 2020 (de acuerdo con el estado de desarrollo, mineral e inversión)

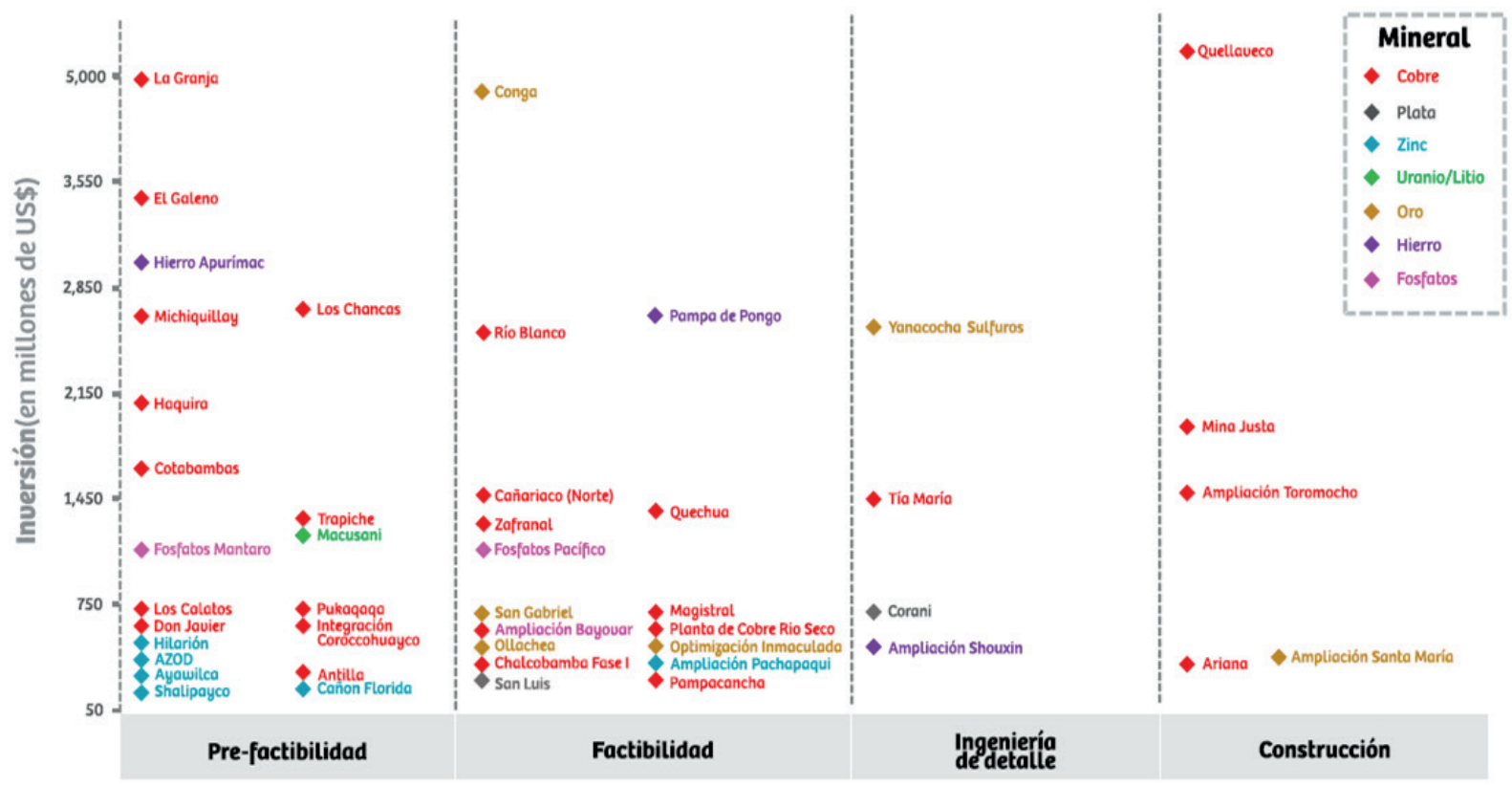

Elaboración: Dirección General de Promoción y Sostenibilidad Minera - Equipo de Estudios Económicos 
Tabla 1

Variación de productos agroindustriales del 2019 y 2020

\begin{tabular}{|c|c|c|c|c|c|}
\hline Ranking & Producto & 2019* & $2020 *$ & Var.\%20/19 & Part.\% \\
\hline 1 & Paltas frescas o secas & 749 & 756 & $0.9 \%$ & $13.0 \%$ \\
\hline 2 & Arándanos frescos & 560 & 742 & $32.5 \%$ & $12.8 \%$ \\
\hline 3 & Uvas frescas & 443 & 510 & $15.1 \%$ & $8.8 \%$ \\
\hline 4 & Café & 463 & 475 & $2.6 \%$ & $8.2 \%$ \\
\hline 5 & Espárragos frescos & 306 & 290 & $-5.2 \%$ & $5.0 \%$ \\
\hline 6 & Mangos frescos & 186 & 225 & $21.0 \%$ & $3.9 \%$ \\
\hline 7 & Plátano fresco & 128 & 127 & $-0.8 \%$ & $2.2 \%$ \\
\hline 8 & Cacao en grano & 120 & 113 & $-5.8 \%$ & $1.9 \%$ \\
\hline 9 & Quinua & 111 & 104 & $-6.3 \%$ & $1.8 \%$ \\
\hline \multirow[t]{3}{*}{10} & Alcachofas & 65 & 74 & $13.8 \%$ & $1.3 \%$ \\
\hline & Resto & 2338 & 2379 & $1.8 \%$ & $41.1 \%$ \\
\hline & Total & 5469 & 5795 & $6.0 \%$ & $100.0 \%$ \\
\hline
\end{tabular}

Nota. $\left(^{*}\right)$ Meses de enero a octubre, adaptado de Superintendencia Nacional de Aduanas y de Administración Tributaria (SUNAT)

y uso sustentable de los recursos marinos en altamar.

\section{La Planificación y Presupuesto Nacional}

El Perú no cuenta con un Plan de Desarrollo a corto, mediano y largo plazo aprobado e implementado para sus sectores productivos y de servicios, situación que genera inestabilidad gubernamental y distorsiones en su desarrollo.

Las entidades gubernamentales formulan sus Planes Estratégicos en función a sus sectores en forma desarticulada del interés nacional. Existe un divorcio entre la planificación y el presupuesto nacional.

\section{El Sistema Financiero}

Está conformado por las Instituciones bancarias, financieras de derecho público o privado autorizadas por la Superintendencia de Banca y Seguro, que captan depósitos como los bancos, empresas financieras, cajas municipales de ahorro y crédito, cajas rurales y el Banco de la Nación.

\section{Sector Tecnológico y las TIC}

En el 2020 se aumentó el uso de plataformas y herramientas tecnológicas, hubo un crecimiento en aplicaciones de e-commerce y pagos online, se tuvieron que reclamar mejoras en su conectividad en casa para acceder a servicios de educación a distancia.
Se desarrollan mejores aplicaciones para la cadena de suministros y logística para el seguimiento en el delivery de las mercancías y el control del stock, se volcaron masivamente al uso de dispositivos móviles, tabletas o laptops, y sus aplicativos.

\section{Medio Ambiente}

El medio ambiente natural comprende componentes físicos, como aire, temperatura, relieve, suelos y el agua, así como componentes vivos como las plantas, animales y microorganismos.

\section{Corrupción en el Perú}

Es definida por Transparencia Internacional como el "abuso de una posición pública para obtener un beneficio privado" (Ugaz, 2018, p.22). La corrupción es el problema más grave que enfrenta el país durante muchos años, y que genera efectos perjudiciales especialmente a los más pobres.

Según la Encuesta Nacional de Valores y Ciudadanía hecha por el Proyecto Especial Bicentenario (2020), el 70\% de los peruanos considera que la corrupción es el principal problema del país y señala específicamente al Poder Judicial, el Congreso de la República y la Policía Nacional como las tres instituciones más corruptas del país. La mayoría de los expresidentes estuvieron involucrados en el caso Lava Jato y en el Poder Judicial están los llamados Cuellos Blancos. 


\section{CONCLUSIONES}

Los proyectos de investigación en las tesis de post grado de la Ciencias Administrativas en el capítulo del planteamiento del problema deben recoger el análisis del macro entorno nacional e internacional en la parte que le corresponda al tema de la investigación por su importancia y efectos directos e indirectos en el desarrollo de su proyecto.

Así mismo, deben tomar en cuenta el análisis de microentorno empresarial con respecto a los clientes, proveedores, competencia, etc.

En los casos de las investigaciones aplicativas debe analizarse el entorno empresarial, que comprende la gestión, las áreas estratégicas y los recursos empresariales.

Con estos insumos se podrá describir la caracterización del problema empresarial y formular las preguntas, general y específicas.

\section{REFERENCIAS BIBLIOGRÁFICAS}

Comisión Económica para América Latina y el Caribe (2021). Balance Preliminar de las Economías de América Latina y el Caribe. Naciones Unidas, CEPAL. https://www.cepal.org/sites/default/files/ publication/files/46501/S2000990_es.pdf
Dirección General de Promoción y Sostenibilidad Minera (2020). Actualización de la Cartera de Proyectos de Construcción de Mina 2020. Boletín Estadístico Minero. http://www.minem.gob. pe/minem/archivos/file/Mineria/PUBLICACIONES/VARIABLES/2020/BEMOCT2020.pdf

Instituto Nacional de Estadística e Informática (2020). Principales Indicadores Macroeconómicos. https://www.inei.gob.pe/estadisticas/ indice-tematico/economia/

Proyecto Especial Bicentenario (2020). Encuesta Nacional de Valores y Ciudadanía. Datum Internacional, Presidencia del Consejo de Ministros. https://drive.google.com/file/d/16dN_QBJFkoFImLcMfappLIZBdR3CApo0/view

Ugaz Sánchez-Moreno, J. C. (2018). Gran corrupción y derechos humanos [Tesis de Maestría, Pontificia Universidad Católica del Perú]. http://tesis.pucp.edu.pe/repositorio/handle/20.500.12404/13363 
